Logos. Anales del Seminario de Metafísica

ISSN: 1575-6866

https://dx.doi.org/10.5209/asem.73222

\title{
Demasiada felicidad. Sobre la teoría de los afectos en Descartes y Leibniz
}

Vicente Raga Rosaleny ${ }^{1}$

Recibido: 24 de diciembre de 2020 / Aceptado: 26 de mayo de 2021

Resumen. Descartes y Leibniz figuran como pensadores opuestos en el ámbito de la filosofía moderna. Tras un período de influencia inicial, el pensador alemán habría sido un crítico severo de las tesis filosóficas del filósofo francés. Sin embargo, una lectura cuidadosa de las dispersas declaraciones leibnizianas a propósito de los afectos y su dimensión moral nos permitirán hacernos cargo de la distancia y cercanía entre el pensamiento de ambos. Para Descartes la felicidad está ligada a la voluntad, mientras que Leibniz será más fiel a la tradición intelectualista aristotélica, aunque su concepción de las emociones es, en muchos sentidos, asombrosamente similar.

Palabras clave: afecto; dualismo; felicidad; placer; tendencia.

\section{[en] Too much Happiness. On the Affections Theory in Descartes and Leibniz}

Abstract. Descartes and Leibniz appear as opposing thinkers in Early Modern philosophy studies. After a period of initial influence, the German thinker would have been a severe critic of the philosophical theses of the French philosopher. However, a careful reading of Leibniz's scattered statements about affects and their moral dimension will enable us to grasp the distance and closeness between the thinking of both authors. For Descartes, happiness is linked to the will, while Leibniz will be more faithful to the Aristotelian intellectualist tradition, although his conception of emotions is, in many ways, amazingly similar.

Keywords: affection; dualism; happiness; pleasure; tendency.

Sumario: 1. Introducción; 2. El problema de la interacción causal: el dualismo y sus descontentos; 3. El tiempo de los afectos: Leibniz y la teleología de las emociones; 4. Demasiada felicidad: Descartes y Leibniz, tan lejos y tan cerca; 5. Conclusión; 6. Referencias bibliográficas.

Cómo citar: Raga Rosaleny, V. (2021) “Demasiada felicidad. Sobre la teoría de los afectos en Descartes y Leibniz”, en Logos. Anales del Seminario de Metafisica 54 (2), 349-364.

\footnotetext{
1 Departament de Filosofia

Facultat de Filosofia i Ciències de l'Educació

Universitat de València

vicente.raga@uv.es
} 


\section{Introducción}

La interpretación usual en los manuales de historia de la filosofía moderna, y entre los especialistas en general, es la de que el pensador francés René Descartes (15961650) y el alemán Gottfried Wilhelm Leibniz (1646-1716) mantienen una relación de oposición y crítica. ${ }^{2}$ Descartes, opuesto a la escolástica aristotélica imperante en las universidades de su tiempo, se convirtió poco después de su muerte en uno de los principales filósofos naturales modernos, y Leibniz pasaría de recibir la influencia del pensamiento cartesiano durante su formación temprana, a discutir y oponerse a las tesis centrales del pensador francés y sus seguidores. ${ }^{3}$

Quizá una de las nociones tradicionalmente atribuidas a Descartes que más oposición despertó en Leibniz (igual que lo había hecho en Hobbes, Gassendi o Spinoza, por citar algunos precedentes notables) fue la del dualismo racional. ${ }^{4}$ Leibniz suele mencionarse como uno de los que contribuyó de manera decisiva a señalar los problemas presentes en la interacción causal entre cuerpo y mente, derivados de la tesis dualista cartesiana, y su monadología puede ser vista como una solución a las dificultades de ésta. Sin embargo, en este artículo pretendo centrarme en un aspecto poco estudiado de la relación entre Descartes y Leibniz, que nos permitirá calibrar de manera más precisa las propuestas de ambos pensadores.

En concreto, atenderé a la "teoría" de los afectos de Leibniz y sus consecuencias éticas, especialmente en relación con un problema clásico, el de la felicidad. Ciertamente Leibniz dedicó poco espacio en sus abundantes escritos al tema de las emociones, a diferencia de lo que podemos observar en el caso de Descartes o de Baruch Spinoza (1632-1677). Apenas dos capítulos de sus Nuevos ensayos sobre el entendimiento humano (1704) parecerían constituir todo su legado intelectual al respecto. Sin embargo, con anterioridad elaboró un manuscrito, titulado De affectibus (1679), que quedó inédito. ${ }^{5}$ A partir de este interesante texto, redactado tras la lectura tanto de los textos de Spinoza como, especialmente, de Las pasiones del alma (1649) de Descartes, y de las escasas páginas de los Nuevos ensayos dedicadas a tal asunto, es posible derivar un esbozo de teoría de los afectos que contrasta y, a la vez, se relaciona, con la cartesiana.

2 Entre otros, puede consultarse, Abbagnano, N.: Historia de la filosofía, vol. 2, trad. de Juan Estelrich y Jorge Pérez, Barcelona, Hora, 1994, pp. 261ss; Belaval, Y.: Leibniz, Critique de Descartes, Paris, Gallimard, 1960 , pp. 138ss o Reale, G. y Antiseri, D.: Historia del pensamiento filosófico y científico, vol. 2, trad. de Juan Andrés Iglesias, Barcelona, Herder, 1992, pp. 388ss, aunque la bibliografía al respecto es abundante, y el acuerdo interpretativo generalizado.

3 Como hizo también, entre otros, Newton, $c f$. Gaukroger, S.: Descartes. An Intellectual Biography, Oxford, Clarendon Press, 1995, p. 383 y Gaukroger, S.: Descartes 'System of Natural Philosophy, Cambridge, Cambridge University Press, 2002, p. 35.

$4 \quad C f$. Descartes, R.: Euvres complètes, Paris, Vrin, 1996, VII, p. 173; Descartes, R.: Meditaciones metafísicas, trad. de Vidal Peña, Oviedo, KrK, 2005, p. 388; Descartes, R.: Euvres complètes, op. cit., VII, pp. 343-344; Descartes, R.: Meditaciones metafisicas, op. cit., p. 668 y Spinoza, B.: Spinoza Opera, vol. 2, Heidelberg, Carl Winters Universitätsbuchhandlung, 1972, II, p. 52; Spinoza, B.: Ética demostrada según el orden geométrico, trad. de Vidal Peña, Madrid, Tecnos, 2007, I, Proposición X, Escolio, p. 76, respectivamente.

5 En el caso de Leibniz citaremos sus textos principalmente a partir de la edición de la Academia alemana de la ciencia, abreviada como "A", con las traducciones españolas cuando proceda debidamente referenciadas (si no existe traducción, como en el caso del De affectibus, ha de entenderse que las versiones al español serán nuestras). Para Descartes, las citas las tomaremos de las obras completas en la edición de Adam y Tannery, abreviada como "AT", con la correspondiente referencia de la traducción española. 


\section{El problema de la interacción causal: el dualismo y sus descontentos}

Sin duda, el destino del pensamiento cartesiano ha sido tan azaroso como el de su cuerpo, si el primero terminó parcialmente desmembrado, con el cráneo por un lado y los restantes huesos por otro, ${ }^{6}$ no menos tergiversaciones podrían haber sufrido sus ideas. Ya en vida, el propio Descartes se lamentaba en este sentido, de manera un tanto cómica, ante su corresponsal y amigo Chanut: "Un tal padre Bourdin creyó tener una buena razón para acusarme de ser escéptico porque había intentado refutar el escepticismo; y cierto pastor trató de demostrar que era ateo sin dar otra razón que el hecho de que había pretendido probar la existencia de Dios".?

$\mathrm{Y}$ es que, si hay una noción clave en las interpretaciones del legado cartesiano que haya mantenido intacta su vigencia desde los tiempos de Descartes hasta nuestros días esa es precisamente la de sustancia. Sin embargo, este concepto era también central para la escolástica aristotélica que predominaba en las universidades en tiempos del pensador francés, y Descartes se oponía a la versión del aristotelismo brindada por sus intérpretes cristianos. ${ }^{8}$

Sea como fuere, parece existir un acuerdo unánime al respecto, y no es difícil encontrar abundantes pasajes en las principales obras de Descartes, del Discurso del método a las Pasiones del alma, en los que el pensador francés se compromete con una concepción metafísica de acuerdo con la cual el mundo se reduciría a dos sustancias principales, irreductibles entre sí. Dichas sustancias, de las que, por cierto, sólo conocemos sus atributos principales, eran la res extensa y la res cogitans, esto es, la sustancia extensa y la pensante. De la primera cabe decir que se concreta en cuerpos, indefinidamente divisibles por su carácter compuesto. De la segunda puede decirse justo lo contrario: la mente sería simple, por ello mismo indivisible, y en ese orden de ideas, esencialmente una en cada instancia de la sustancia pensante, es decir, por cada individuo concreto.

En el caso de los animales no humanos, así como en el de los objetos inanimados, parece claro para el pensamiento cartesiano, aunque no exento de polémica, que los ejemplares tan sólo están dotados de extensión. ${ }^{9}$ En el caso de los seres

\footnotetext{
Shorto, R.: Los huesos de Descartes, trad. de Claudia Conde, Barcelona, Planeta, 2011, p. 164.

AT IV, p. 536.

Por ejemplo, explícitamente pidió a su polémico seguidor Regius que evitase dicha noción escolástica en una carta de enero de 1642, $c f$. AT III, p. 492. Aunque, ha de señalarse que la relación entre el pensamiento aristotélico y el cartesiano es compleja, pues como diversos autores han indicado (cf. Marion, J.-L.: Sobre la ontología gris de Descartes, trad. Alejandro García Mayo, Madrid, Escolar y Mayo Editores, 2008, pp. 221ss), la innovación mediante la que Descartes piensa la modernidad de la metafísica emerge de un continuo con la tradición escolástico-aristotélica, en el que muchos temas y tópicos han de mantenerse para que sean posibles las inversiones y críticas. Agradezco en este punto, así como en otros, los comentarios de los evaluadores anónimos de la revista, que me han permitido mejorar sustantivamente una versión previa de este texto.

9 Ya en la quinta parte del Discurso del Método, en la carta a Reneri para Pollot de abril o mayo de 1638 (cf. AT II, pp. 39-41), y en las respuestas al cuarto conjunto de objeciones a las Meditaciones metafísicas, así como en la carta al Marques de Newcastle (1592-1676) del 23 de noviembre de 1646 ( $c f$. AT IV, pp. 573-576) o en la carta al pensador Henry More (1614-1687) del 5 de febrero de 1649 (cf. AT V, pp. 275-279; González Recio, J. L. (ed.): La correspondencia Descartes-Henry More, trad. de Dolores Escarpa e Irene Pajón, Madrid, Antígona, 2011, pp. 75s) con el recurso al modelo del autómata Descartes deja abolida la distinción entre lo inerte y lo vivo. Sin embargo, como con tantos otros puntos cruciales del pensamiento cartesiano, tales aseveraciones han de ser matizadas, y no es claro que el pensador francés suscribiese por completo la tesis del animal-máquina (término que, por cierto, jamás empleó). Al respecto, pueden consultarse tanto la carta dirigida a Plempius para Fromondius del 3 de octubre de 1637 ( $c f$. AT I, pp. 413-416) como una misiva a Buitendijck de 1643 ( $c f$. AT IV, p. 65), en las que no se excluye la posibilidad de un alma animal.
} 
humanos las cosas son un poco distintas, porque al menos en la interpretación más inmediata de los textos de Descartes el ser humano parece un compuesto, accidental o contingentemente unido, de cuerpo extenso y pensamiento. ${ }^{10} \mathrm{Y}$ el problema principal, que no se plantea para los animales no humanos o, por mencionar entes que ejemplificarían para el cartesianismo una res cogitans pura, los ángeles, es el de cómo se relacionan dos sustancias radicalmente diferentes y hasta opuestas.

Es decir, parece claro que, entre los elementos compuestos de sustancia extensa las relaciones son simples: se dan choques entre los corpúsculos que constituyen el universo material inerte cartesiano, carente de vacío, y puede hablarse de una interacción causal transparente entre los distintos elementos. Pero, ¿qué lugar ocupa la mente en un mundo mecanicista? La res cogitans no puede estar relacionada causalmente con la sustancia extensa, porque la causalidad es una función de la extensión, y la mente carece de este atributo por definición. Y, a la inversa, no queda claro cómo se relacionaría la mente inextensa con un mundo físico en el que, por decirlo en términos actuales, impera la clausura causal. Esto es, donde al pensamiento no le queda por cumplir papel alguno que no cumpla ya la materia. Así, la interacción entre cuerpo y mente, por culpa de una concepción metafísica deficiente, se torna misteriosa $y$, en última instancia, inexplicable. ${ }^{11}$

Sin embargo, esta distinción real entre cuerpo y mente, que tantas críticas suscitó desde la época de Descartes en adelante no surgió de la nada, aislada como un aerolito filosófico, ni tampoco se explica simplemente acudiendo a la necesidad de acomodar los avances de la ciencia de la época cartesiana con los compromisos cristianos más ortodoxos (aunque, sin duda, la preocupación por salvaguardar la libertad, la moralidad o la doctrina de la inmortalidad en un mundo mecanicista pudieron tener su peso en la argumentación de Descartes). En lugar de eso, es más bien, de nuevo, la oposición del pensador francés a las doctrinas dominantes en las universidades de su tiempo la que puede darnos una clave interpretativa esclarecedora.

No en vano se debió a la influencia aristotélica el que hasta bien entrado el Renacimiento se concibiera el alma como una entidad tripartita. De esta forma, siguiendo lo enunciado por Aristóteles en sus textos dedicados al estudio de los animales y en el consagrado al alma, los escolásticos entendieron que el mundo animado podía dividirse en tres esferas: la de las plantas, regidas por un alma vegetativa dedicada al cuidado de la alimentación, la sensación y, en suma, todo lo corpóreo. Luego la de los animales, que junto con el primer tipo de alma tendrían otra, la sensitiva, consagrada a regir la memoria, la imaginación o el deseo. Y, por último, en esta scala naturae se encontraría el ser humano, que a las dos almas previas añadiría la racional, que ordena el intelecto y todas las funciones superiores. $\mathrm{Y}$ aunque el mundo inanimado no estaría completamente separado de las distintas especies de seres vivos, claramente un artefacto, o un autómata, lo mismo que una piedra, no se regirían por idénticos principios que los entes animados. ${ }^{12}$

10 Así lo expresa claramente, por ejemplo, en Meditaciones metafísicas: “[...] soy sólo una cosa que piensa -y no extensa-, y, por otra parte, tengo una idea distinta del cuerpo, en cuanto que él es sólo una cosa extensa -y no pensante- [...]" (AT VII, p. 78; Descartes, R.: Meditaciones metafisicas, op. cit., p. 232).

11 Damasio, A. R.: Descartes' error. Emotion, Reason, and the Human Brain, New York, Avon Books, 1995 , p. 247; Williams, B.: Descartes: The Project of Pure Enquiry, London, Routledge, 2005, p. 273.

12 Véase, por ejemplo, Aristóteles: Partes de los animales. Marcha de los animales. Movimiento de los animales, trad. de Elvira Jiménez y Almudena Alonso, Madrid, Gredos, 2000 y Aristóteles: Acerca del alma, trad. de Tomás Calvo, Madrid, Gredos, 1978, para una exposición detallada de este modelo explicativo que extendió su 
Pero, para Descartes los poderes del alma vegetativa y de la sensitiva, o por decirlo de otro modo, de las partes anímicas dedicadas a regir las funciones inferiores del ser humano, o de los restantes animales y plantas, no tenían cabida en un mundo reducido a dos sustancias. Por eso, dichas actividades se las asignaría a los cuerpos en que se concreta la res extensa, convertidos ahora sí en autómatas y, por ello mismo, dirigidos por las mismas leyes derivadas de los choques de los corpúsculos de que se componen homogéneamente las plantas, los animales no humanos y el cuerpo del hombre, las rocas o las ruedecillas de un reloj de pulsera. ${ }^{13}$

Mientras tanto, el alma, reducida ahora a su dimensión racional, en tanto que simple, sin partes y ya no jerárquicamente dividida, únicamente tendría un poder, el de pensar. Sin embargo, que Descartes desdeñase el modelo psicofisiológico del aristotelismo escolástico por su falta de claridad y distinción, no significaba reducir la mente a la mera función racional. En efecto, el entendimiento, y la voluntad, son los dos modos activos principales de la res cogitans, pero el cogito no sólo piensa o quiere, sino que también siente. Así pues, parte de lo que constituía el alma sensitiva aristotélica terminaría recalando en la mente cartesiana, con especial mención de lo que Descartes denomina las pasiones, modo pasivo de lo mental cuyo despliegue requiere, en su mayor parte, de una decidida unión entre mente y cuerpo. ${ }^{14}$

No obstante, la contextualización del pensamiento de Descartes en este punto concreto no parece favorecer la causa del cartesianismo. Después de todo, el hecho de que reaccionase contra la oscuridad de las explicaciones escolásticas, con sus misteriosas formas activas sustanciales, en nada aclaraba la manera en que podían reconectarse de manera adecuada el cuerpo y la mente humanas una vez separadas. Y la referencia a las pasiones como aquel modo de lo mental que requiere de nuestros cuerpos particulares para expresarse no contribuía precisamente a esclarecer el problema de la relación entre ambas sustancias. Peor aún, las explicaciones fisiológicas detalladas, con las pasiones cartesianas como un resultado del fluido corporal que recorre nuestros nervios, pronto resultaron claramente insatisfactorias: ${ }^{15}$ ni los espíritus animales podían interactuar causalmente con el modo mental de las emociones, ni éstas, en tanto que atributo de la sustancia anímica, con una innegable dimensión representacional, parecían tener forma de afectar al cuerpo humano.

El pensador alemán Leibniz estaría probablemente de acuerdo con esta conclusión, puesto que no en vano fue un crítico destacado del dualismo atribuido a Descartes. Para él, mente y mundo no se podían relacionar como parecía asumirlo el filósofo francés, en virtud de algún tipo de interacción entre sustancias absolutamente diversas y separadas. De ahí su famosa objeción a la posibilidad de que la mente pueda modificar la dirección de los espíritus animales ${ }^{16}$ o por decirlo de otro modo, su crítica a la posibilidad de que exista una modificación de lo corpóreo en virtud de una intervención directa de lo mental, bien sea por un acto de voluntad, bien por una

influencia desde la baja Edad Media hasta los tiempos de Descartes.

13 Cf. AT XI, p. 202; Descartes, R.: El tratado del hombre, trad. de Guillermo Quintás, Madrid, Alianza, 1990, p. 109.

14 Cf. AT XI, pp. 349-350; Descartes. R.: Las pasiones del alma, trad. de José Antonio Martínez y Pilar Andrade, Madrid, Tecnos, 2006, § 28.

15 Como evidencia de manera demoledora, por ejemplo, la correspondencia de Isabel de Bohemia con Descartes, cf. AT III, p. 661; Descartes, R.: Correspondencia con Isabel de Bohemia y otras cartas, trad. de Maite Gallego, Barcelona, Alba, 1999, pp. 25-26.

16 Cf. Leibniz, G. W.: Monadología, trad. de Julián Velarde, Oviedo, Pentalfa, 1981, § 80. 
pasiva influencia emocional. La manera en que se relacionan la acción y la pasión, tanto en general como en los afectos en particular, debía concebirse de otro modo, y a formular una propuesta aparentemente alternativa se dedicaría Leibniz en algunos textos dispersos pero muy relevantes.

\section{El tiempo de los afectos: Leibniz y la teleología de las emociones}

Como se indicó previamente, el interés de Leibniz por lo que usualmente denomina los afectos (siguiendo aquí la terminología de Spinoza), no es precisamente tardío. Ya en el manuscrito De affectibus, que el pensador alemán inició como una serie de notas a Las pasiones del alma de Descartes, se abordan estos de manera decidida, empezando por dar unas definiciones que mantendrá de manera coherente en los Nuevos ensayos sobre el entendimiento.

Así, para Leibniz "el afecto es una ocupación del alma que surge de la opinión sobre lo bueno y lo malo". ${ }^{17}$ Es decir, que el afecto brota de las opiniones o juicios sobre lo que resulta placentero o desagradable de una manera espontánea, y es una tendencia que, finalmente, nos lleva a actuar en un sentido u otro, para alcanzar el objeto de nuestras emociones o para evitarlo. En ese sentido, y siguiendo de nuevo a Spinoza, puede decirse que el afecto se constituye como la suma de una representación y una inclinación o conatus. Y, cabe añadir, nada de lo que se indica hasta aquí se opone a las ideas de Descartes al respecto. ${ }^{18}$

De hecho, aunque en el manuscrito se establece una doble posibilidad, que los afectos procedan de la razón o de las percepciones, tampoco esto se opone a la concepción cartesiana, para el que las "pasiones intelectuales" provendrían, en cierto sentido, del entendimiento, y las restantes tendrían que ver mucho más con la sensibilidad. Hasta este punto, pues, Leibniz no parecería disentir del cartesianismo, ya que ambos concuerdan en el carácter cognitivo y conativo de las emociones, para los dos autores éstas nos permitirían hacernos cargo del mundo y actuar en consecuencia.

Sin embargo, un poco más adelante el pensador germano es mucho más explícito, al aseverar que "los afectos son percepciones, sensaciones o trastornos del alma, que se remiten a ella en particular [...] sin que nadie conozca una causa próxima a la que puedan remitirse". ${ }^{19} \mathrm{Y}$ es que aquí, haciéndose eco de la definición cartesiana de las pasiones, al mismo tiempo que reconoce en gran medida la validez de las descripciones de Descartes también se distancia de ellas de manera ostensible. Para el pensador francés las pasiones son igualmente percepciones o emociones que refieren al alma, pero que se producen gracias al movimiento de los espíritus animales. Tienen, pues, un trasfondo fisiológico necesario, con los problemas de la interacción causal que ya conocemos, al que Leibniz hace escasa mención y siempre en tono crítico.

La teoría leibniziana de los afectos se aleja pues de la dimensión corpórea de las pasiones cartesianas ya desde un principio, aunque asume su carácter representacional y motivacional plenamente. Es una propuesta, la de Leibniz,

\footnotetext{
A VI. 4, p. 1412.

Cf. AT IV, p. 284; Descartes, R.: Correspondencia con Isabel de Bohemia y otras cartas, op. cit., p. 96. A VI, 4, p. 1418.
} 
marcadamente psicológica y, en esa misma línea, sus reflexiones metafísicas y epistémicas posteriores serán coherentes con dicho punto de partida. No es que no exista una dimensión fisiológica para el pensador germano, de hecho, en sus escritos médicos las pasiones también tendrán un espacio reservado, pero en la reflexión propiamente dirigida a los afectos no son las hipótesis cartesianas aquello a lo que se apela, sino otra concepción muy relevante para Leibniz, a saber, la de expresión. ${ }^{20}$

Y es que tanto en el caso de Descartes como en el de Leibniz los afectos presentan un dinamismo interno. Claramente, en el planteamiento cartesiano el paso de la pasividad a la actividad, y viceversa, ya no estaba ligado a la existencia de diversas partes del alma, jerárquicamente dispuestas, como lo pretendía la escolástica aristotélica. En lugar de ello, la tensión se establece entre las pasivas emociones, instanciadas gracias al cuerpo, y la voluntad activa, cuya sede sería la mente. Pero esto no vale para el pensador germano, quien explícitamente indica en varios pasajes de su obra que lo corpóreo nada puede percibir en relación con sus propias acciones y pasiones, al carecer de pensamiento. ${ }^{21}$

Para Leibniz, pues, los afectos son estados o, mejor dicho, tendencias autosuficientes, que se alternan en los papeles activo y pasivo para generar el mencionado dinamismo psicológico. ${ }^{22}$ No hay entonces una relación de interacción o dependencia entre mente y cuerpo, al estilo de la que se le atribuye a Descartes, sino que la mente se expresa de manera espontánea, ciertamente bajo la forma de representaciones de lo que le sucede al cuerpo y su entorno, pero no por influjo directo de éste. ${ }^{23}$ Igual que un mapa traspone de manera simbólica los accidentes geográficos de un espacio, sin que haya un vínculo de interdependencia con la orografía del paisaje, los afectos emergerían en la mente del sujeto leibniziano representando en la simplicidad de ésta lo externo y compuesto, aquello que es ajeno a la mónada. ${ }^{24}$

20 Cf. Vargas, E.: "Perceiving machines: Leibniz's teleological approach to perception”, en J. E. H. Smith y O. Nachtomy (eds.), Machines of nature and corporeal substances in Leibniz, Dordrecht, Springer, 2011, p. 178.

21 Cf. A VI. 2, p. 266.

$22 C f$. Di Bella, S.: "Le De affectibus leibnizien: de la dynamique des passions à la constitution de la substance individuelle", en P.-F. Moreau (ed.), Les passions à l'âge classique. Théorie et critiques des passions, vol. 2, Paris, Presses universitaires de France, 2006, p. 193.

23 Cf. A VI. 4, p. 1411.

24 Vale la pena señalar que la formulación más temprana de la noción de expresión, y probablemente también la más clara, la encontramos en el breve opúsculo, de apenas dos páginas, titulado Quid sit idea, cf. A VI. 4, pp. 1369-1370. En este pequeño conjunto de anotaciones de 1678 señala Leibniz, lo siguiente: "Se dice que una cosa expresa otra cuando tiene relaciones [habitudines] que corresponden a las relaciones de la cosa expresada", y luego pasa a mencionar una serie de ejemplos de lo que tiene en mente al definir así esta noción: "Por ejemplo, el modelo de una máquina expresa la máquina misma, la delineación en perspectiva de un cuerpo expresa el sólido, las frases expresan los pensamientos y las verdades [...]". Aunque es motivo de debate qué quiere decir exactamente Leibniz, y así algunos entienden que la expresión establece una relación biunívoca entre los elementos singulares de un objeto y los de otro ( $c f$. Kulstad, M.: "Leibniz's concept of expression", Studia Leibnitiana, 11/1, 1977, p. 69), mientras que otros señalan como necesaria la existencia de una analogía entre las relaciones inherentes a ambos objetos (cf. Swoyer, Ch.: "Leibnizian expression", Journal of the history of philosophy, 33/1, 1995, p. 77).

Sea como fuere, ambas posturas son compatibles, puesto que lo relevante es que exista una cierta analogía entre los dos extremos, teniendo en cuenta tanto los elementos singulares como sus relaciones, para que seamos capaces de movernos entre lo expresado y su expresión. Esto es posible, de acuerdo con Leibniz, gracias a una suerte de correspondencia estructural, que puede surgir de la semejanza natural, como en el caso de la relación entre un territorio y su mapa, o de la pura convención, como en el nexo que cabe establecer entre una frase y el pensamiento expresa. Adicionalmente, para los objetivos de este artículo la importancia clave de la noción de expresión reside en que permite establecer una relación entre mente y cuerpo sin apelar al problemático interaccionismo atribuido a Descartes y, como se indica en Quid sit idea, inferir conocimientos de la cosa 
Finalmente, y como posible solución al dualismo cartesiano, en la Monadología se propuso la armonía preestablecida, como recurso para dar cuenta de la innegable coincidencia entre lo que sucede en nuestros cuerpos y nuestros procesos mentales. ${ }^{25}$ En efecto, no sería la problemática interacción entre dos sustancias distintas e irreconciliables la que explicaría que cuando tengo miedo de un animal salvaje corra en dirección contraria, ni tampoco habría que aludir a misteriosas, y puntuales, intervenciones divinas con ocasión de dichos eventos, como proponía algún seguidor de Descartes, especialmente el padre Malebranche. En lugar de ello, los afectos coincidirían exactamente con las conductas de acuerdo con un orden establecido desde el principio de los tiempos por la divinidad, sin restar con ello espontaneidad alguna a su emergencia en la mente humana.

Pero entonces, si son independientes del cuerpo, aunque armónicamente correspondan a las conductas de éste, ¿de dónde surgen los afectos y en qué consiste concretamente su dinamismo? Si atendemos ahora a las referencias que el maduro Leibniz hace de las emociones, y específicamente a lo que indica en los Nuevos ensayos, nos encontramos con que, a diferencia de lo que parece suceder en el caso de Descartes, para el que la mente sería transparente, en el caso leibniziano las cosas son un tanto más turbias, aunque en un sentido positivo.

Cabe pues decir que Leibniz, que tantas sugerencias innovadoras proporcionó en el campo de las matemáticas y de la física, no dejó de ser también pionero en el ámbito psicológico. Y es que los afectos en la interpretación leibniziana surgirían de una dimensión de la mente apenas explorada todavía en aquellos tiempos, y que debería esperar prácticamente hasta nuestros días para ser reivindicada, el ámbito de lo inconsciente. Pues si bien es cierto que el origen de las emociones, como ya dijimos, ha de buscarse en las opiniones sobre lo placentero y displacentero, antes de eso el afecto se va gestando a partir de la inquietud que suscitan lo que Leibniz llama las "pequeñas percepciones insensibles". ${ }^{26}$

Leibniz hace referencia a éstas en diversos pasajes, pero hay uno justamente famoso, en el que empleando una metáfora las compara con las innumerables olas que chocan con la costa. Por separado no captamos ninguna de ellas, pero es la suma total de ese vaivén marítimo la que produce el característico rumor del oleaje que podemos escuchar siempre que nos asomemos al borde de un mar en calma. ${ }^{27}$

Ciertamente las pequeñas olas las percibimos sin caer en cuenta de ello, y permanecen activas, diríamos hoy, en el inconsciente, constituyendo la base de nuestras afecciones. Así, nuestra vida mental se nutre de las innumerables percepciones que despierta nuestro entorno, indiscernibles de manera individual pero poderosamente presentes de forma colectiva. En el caso de los afectos, lo que inicia siendo una serie de mínimas inclinaciones que nos provocan cierta inquietud, se va decantando hasta llegar a las tendencias de pensamiento que nos conducen a la acción. ${ }^{28}$ Se puede hablar así de un proceso, que como veremos, está teleológicamente guiado desde la perspectiva leibniziana, por medio del cual nuestras afecciones empiezan en lo

expresada, el cuerpo y sus estímulos externos en este caso, a partir del análisis de las relaciones inherentes a su expresión, las pasiones o afectos.

25 Cf. Leibniz, G. W.: Monadología, op. cit., § 49.

${ }_{26}$ Cf. A VI. 6, pp. 115-116; Leibniz, G. W.: Nuevos ensayos sobre el entendimiento, trad. de Juan R. Goberna, Madrid, Akal, 2016, p. 167.

27 Cf. A VI. 6, p. 54; Leibniz, G. W.: Nuevos ensayos sobre el entendimiento, op. cit., p. 88.

28 Cf. A VI. 6, pp. 164-165; Leibniz, G. W.: Nuevos ensayos sobre el entendimiento, op. cit., p. 235. 
inconsciente y terminan en inclinaciones sensibles, que pueden aliarse u obstaculizar las tendencias reflexivas. ${ }^{29}$ Estas afecciones surgen así de las percepciones insensibles, unidas a una opinión sobre lo bueno y lo malo, en ese sentido son representacionales, y apuntan a un objeto, son intencionales, es decir, que se inclinan o tienden a algo, o mejor, modifican las series de nuestros pensamientos dirigiéndonos a la acción. ${ }^{30}$

Pero, a diferencia de lo que, como veremos, sucede en el caso de Descartes, no todo es tan positivo en esta caracterización de los afectos. Pues estos están ligados al placer y al dolor, en efecto, y al aseverar tal cosa Leibniz sigue la idea aristotélica de que el ser humano apunta siempre al bien entendido como fuente de placer. ${ }^{31}$ Pero como se indica ya desde el De affectibus, y se sigue sosteniendo en los Nuevos ensayos, no todos los placeres son iguales y, en concreto los que proceden de las tendencias afectivas suelen ser confusos, a diferencia de aquellos que se ligan a la razón. ${ }^{32}$ Más aún, en el caso de los primeros la dimensión temporal es la del presente puntual, mientras que, en los segundos (aquellos que, por cierto, Leibniz liga a la felicidad), se apunta al futuro y, especialmente, a la perduración estable de lo placentero en el curso del tiempo. ${ }^{33}$

Y aquí es precisamente donde se deja ver el carácter teleológico de la "psicología" leibniziana, puesto que el dinamismo de la vida mental no es azaroso, sino que esa alternancia expresiva de actividad y pasividad, que empuja las series de pensamientos, tiene una meta determinada, la de la perfección humanamente posible. Así, para el pensador alemán los pensamientos ven modificada su dirección de acuerdo con el influjo de los afectos, pero cuando estos se convierten en obstáculos, por su carácter presentista, entra en juego la razón, que nos proporciona la beatitud terrenal si nos dejamos llevar por ella.

En conclusión, pues, queda claro que, pese al escaso espacio dedicado por Leibniz a la reflexión sobre los afectos, estos ocupan un lugar central en su concepción de la vida mental del ser humano. Aún más, puede decirse que la manera en que el pensador germano concibe los afectos parece contrastar claramente con la propuesta cartesiana, o que, al menos, se propone solucionar los principales problemas del dualismo atribuido a Descartes. Y junto con esto, al hilo de lo expuesto resulta obvia la conexión entre psicología y moral, puesto que el análisis leibniziano de los afectos no sólo da cuenta de una concepción dinámica de la mente, frente al supuesto esquematismo y rigidez de la propuesta cartesiana, sino que además propone, muy en línea con el ideal ilustrado, que el control de la razón sería la solución a los posibles desordenes pasionales.

\section{Demasiada felicidad: Descartes y Leibniz, tan lejos y tan cerca}

Si la descripción tradicional del pensamiento cartesiano fuese indiscutiblemente correcta, especialmente en relación con las atribuciones de dualismo mencionadas al

29 Cf. Ebbersmeyer, S.: "Leibniz on the Passions and the Dynamical Dimension of the Human Mind", en S. Ebbersmeyer (ed.), Emotional Minds. The Passions and the Limits of Pure Enquiry in Early Modern Philosophy, Berlin, Boston, De Gruyter, 2012, p. 147.

$30 \quad$ Cf. A VI. 4, p. 1433.

31 Aristóteles: Ética nicomáquea. Ética eudemia, trad. de Julio Pallí, Madrid, Gredos, 1985, 1105b23.

32 Cf. A VI. 4, p. 1420.

33 Cf. A VI. 6, pp. 199-200; Leibniz, G. W.: Nuevos ensayos sobre el entendimiento, op. cit., p. 275. 
inicio de este texto, sin duda la propuesta de Leibniz sería una valiosa alternativa crítica, que además tendría visos de mayor verosimilitud que la interpretación cartesiana de las emociones intelectuales y su enigmática relación con el cuerpo. Sin embargo, en el campo de la historia de la filosofía es posible encontrar cuestionamientos a la lectura usual de Descartes como un dualista racional puro y en especial en relación con su concepción de las pasiones, tomando como punto de partida una lectura más panorámica y contextualizada del pensamiento cartesiano. ${ }^{34}$

$\mathrm{Y}$ es que, es evidente que en el plano metafísico Descartes se compromete con una descripción del mundo dualista y, al menos, con unos atributos sustancialistas, pero ésta no agota todos los ámbitos de interés de dicho filósofo. De hecho, incluso en las Meditaciones metafisicas hay espacio para una comprensión no dualista del ser humano. En concreto, en la sexta meditación se insiste de manera bien llamativa en la diferencia existente entre los demás cuerpos extensos y mi cuerpo, pues de este último no me es dado dudar, además de que su unidad no es contingente, sino que viene determinada por el pensamiento, con el que está como mezclado estrechamente. ${ }^{35}$

Esto quizá suene extraño, y acaso podría interpretarse como una cierta incoherencia por parte del autor francés, que tan claramente había insistido en las primeras meditaciones en la distinción real de las sustancias. Sin embargo, las rotundas declaraciones del autor al respecto de la unión del ser humano no parecen dejar dudas en su posterior correspondencia con la princesa Isabel de Bohemia, siendo estas cartas además una suerte de esbozo del último libro que alcanzaría a escribir, Las pasiones del alma, que nuevamente insiste en la unidad del ser humano. Ese es el caso, por ejemplo, de las nociones primitivas donde se alude precisamente a esa idea de un ser humano uno, al menos desde una perspectiva moral. ${ }^{36} \mathrm{Y}$ eso se evidencia aún más en el modo de pensamiento en el que se entrecruzan con mayor claridad las consideraciones "antropológicas" cartesianas con las cuestiones morales, a saber, con las pasiones.

Efectivamente, al menos desde una perspectiva práctica, en la cotidianeidad de nuestras vidas y en relación con la acción, en un espacio allende de la metafísica y de la ciencia natural, a Descartes le parece claro que la unión corpóreo-mental es innegable. Y, en ese sentido, aunque las pasiones tengan una base fisiológica, que Leibniz no acepta en su descripción de los afectos, el principal obstáculo, la inexplicable interacción entre sustancias distintas queda desdibujado.

No sólo eso, sino que una mayor atención a este modo del pensamiento cartesiano, necesariamente unido al cuerpo, y por ello de carácter obligatoriamente pasivo o receptivo, presenta asombrosas semejanzas con la caracterización de los afectos leibnizianos, una vez dejado de lado el interaccionismo causal entre sustancias. En concreto, precisamente el hecho de que sea un modo inextricablemente unido a la carne abre perspectivas poco atendidas en la concepción cartesiana de lo mental. Y así, en contraste con las interpretaciones usuales, que inciden en la auto-transparencia característica del sujeto pensante, lo que encontramos en las pasiones cartesianas es

34 Véase, por ejemplo, Brown, D. J.: "The Sixth Meditation: Descartes and the Embodied Self", en D. Cunning (ed.), The Cambridge Companion to Descartes' Meditations, Cambridge, Cambridge University Press, 2014, pp. 240-257 o Simmons, A.: "Mind-Body Union and the Limits of Cartesian Metaphysics", Philosophers' Imprint, 17/14, 2017, pp. 1-36, entre otros.

35 Cf. AT VII, p. 74; Descartes, R.: Meditaciones metafisicas, op. cit., pp. 225-226.

36 Cf. AT III, pp. 690ss; Descartes, R.: Correspondencia con Isabel de Bohemia y otras cartas, op. cit., pp. 35ss. 
una cierta oscuridad y un difícil acceso introspectivo para el ser humano apasionado. ${ }^{37}$ Ciertamente, el "inconsciente" cartesiano, compuesto de inclinaciones desconocidas para quien las padece, de ensueños y afecciones a menudo inexplicables, no sería asumible para Leibniz, en la medida en que depende de la estrecha vinculación del modo mental pasional con el cuerpo. ${ }^{38}$ Pero el hecho de que las pasiones dejen paso a la posibilidad del error y la confusión, a la hora de entender a los demás y a uno mismo, así como su origen, a menudo insensible y que por tanto pasa desapercibido al sujeto, inevitablemente recuerda el concepto leibniziano de las "pequeñas percepciones". También las pasiones cartesianas proceden de un "afuera", sin ser notadas hasta que poco a poco se imponen y nos emocionan como ninguna otra percepción logra hacerlo, o eso se desprende de ejemplos como el de la pasión infantil por una niña bizca que Descartes le confiesa a su corresponsal Chanut en una misiva del 6 de junio de 1647, y que terminó convirtiéndose en una fijación pasional hasta que pensó detenidamente sobre ello. ${ }^{39}$

Pero no terminan ahí los puntos de contacto, puesto que, como ya señalamos, en ambos autores las pasiones tienen un carácter representacional y conativo, pudiendo además destacarse ahora que coinciden en su interpretación teleológica de las mismas, cosa que en el marco del mecanicismo cartesiano no puede dejar de resultar sorprendente. En cuanto a lo primero, en efecto, tanto para Leibniz como para Descartes las emociones nos ayudan a trazar un mapa valorativo de nuestro entorno, facilitándonos una relación muy distinta con éste de la que nos permiten las herramientas conceptuales, por ejemplo.

$\mathrm{Y}$ aquí entra en juego el segundo aspecto mencionado, porque las pasiones cartesianas, como los afectos leibnizianos, no sólo nos permiten relacionarnos con

37 Cf. Brown, D. J.: Descartes and the Passionate Mind, Cambridge, Cambridge University Press, 2006, pp. 151152.

38 Véase Rodis-Lewis, G.: Le problème de l'inconscient et le cartésianisme, Paris, Presses universitaires de France, 1950, para un muy interesante estudio al respecto. En cuanto a ejemplos de inclinaciones de carácter proto-inconsciente, puede citarse AT XI, p. 441; Descartes. R.: Las pasiones del alma, op. cit., § 147, como un ejemplo notorio de dichos impulsos confusos y difíciles de percibir.

39 Cf. AT V, p. 57; Descartes, R.: Correspondencia con Isabel de Bohemia y otras cartas, op. cit., p. 252. Es importante notar que la relación entre el "inconsciente" cartesiano y las "pequeñas percepciones" de Leibniz no es ni muchos menos obvia. Después de todo, si algo caracteriza a los variados fenómenos que se agrupan bajo el principal atributo de la res cogitans, el pensamiento, es su carácter consciente (Simmons, A.: "Changing the Cartesian Mind: Leibniz on Sensation, Representation and Consciousness", The Philosophical Review, 110/1, 2001, p. 34). No obstante, en distintos momentos Descartes insiste en que, por ejemplo, las sensaciones son modos confusos de pensamiento, que pueden engañarnos pues "la naturaleza humana, en cuanto compuesta de espíritu y cuerpo, no puede dejar de ser falaz a veces", AT VII, p. 88; Descartes, R.: Meditaciones metafísicas, op. cit., p. 248. Lo mismo sucede con las pasiones, cuya causa última y más próxima está en la "agitación con que los espíritus mueven la pequeña glándula que está en medio del cerebro", AT XI, p. 371; Descartes. R.: Las pasiones del alma, op. cit., §51, pero cuyo origen más lejano son determinadas impresiones fortuitas en el cerebro, que puede causar la misma alma o, más ordinariamente, los objetos externos que estimulan nuestros sentidos y que, por ello, no dejan de prestarse a la confusión, la ignorancia o el error.

En suma, efectivamente, las "pequeñas percepciones" del pensador germano constituyen un reto para uno de los rasgos centralmente atribuido al cartesianismo, la supuesta transparencia de lo mental: "Antes he demostrado que nosotros tenemos siempre una infinidad de pequeñas percepciones, sin que nos apercibamos de ellas. Nunca dejamos de tener percepciones, pero es necesario que estemos a menudo sin apercepciones, como cuando no hay en nosotros percepciones distinguidas", A VI. 6, pp. 162; Leibniz, G. W.: Nuevos ensayos sobre el entendimiento, op. cit., pp. 232. Sin embargo, es posible rastrear, como se ha señalado, la existencia de pensamientos confusos en tanto que representaciones de fenómenos físicos, como una suerte de proto-inconsciente en los textos de Descartes, que anuncian las "pequeñas percepciones", aunque sin la relevancia, ni el aparato metafísico que subyace a la explícitamente polémica propuesta de Leibniz. 
el mundo mediante valoraciones encarnadas, sino que también dirigen nuestras acciones, a través de la modificación del curso de nuestros pensamientos. Las emociones en ambos casos son tendencias, distintas de las voliciones o de otros fenómenos mentales, porque la voluntad o el entendimiento no sienten, pero el ser humano real, el vrai homme cartesiano, experimenta a partir de esas representaciones, de manera turbia y casi incontrolable, las inclinaciones a la acción que Leibniz denomina afectos. ${ }^{40}$

Más aún, esas emociones, que también para Descartes pueden obstaculizar o ayudar a determinadas series de pensamientos, apuntan, igual que en Leibniz, a un objetivo, tienen carácter teleológico, y uno nuevamente congenial con la manera de concebir la finalidad del pensador germano. Por decirlo brevemente, las pasiones cartesianas, como explícitamente señala Descartes en diversos momentos, ${ }^{41}$ tienen como objetivo principal la preservación de nuestra existencia y bienestar en este mundo. Pero eso supone, claramente, la reintroducción de causas finales en un universo mecanicista, cosa con la que Leibniz estaría plenamente de acuerdo y que para el filósofo francés no parece presentar el menor problema, dado que estamos en el campo de la moral y no en el de la filosofía natural o en el de la metafísica.

Por último, cabe señalar que tanto para Descartes como para Leibniz las emociones no deben confundirse con las opiniones, pese a su dimensión cognitiva. Y eso les distancia asimismo de la interpretación usualmente negativa de las pasiones que procede de la tradición estoica. Aunque deudores ambos de los finos análisis de los afectos de un Séneca o un Epicteto, ninguno comparte el ideal de serenidad del sabio estoico, carente de emociones, porque ni para Descartes ni para Leibniz son éstas errores del juicio, es decir, meras opiniones. ${ }^{42}$ Pero si en este punto de nuevo coinciden plenamente, también de aquí surge una de las mayores diferencias entre los dos pensadores.

Así, mientras que, para Descartes, prácticamente todas las pasiones, observadas de cerca, son buenas, ${ }^{43}$ para Leibniz los afectos, como ya comentamos, pueden resultar un serio obstáculo para lograr la felicidad. ${ }^{44}$ En efecto, en ambos casos se

$40 \quad$ Cf. AT VII, p. 81; Descartes, R.: Meditaciones metafisicas, op. cit., p. 237.

${ }^{41}$ Así se lo manifiesta a la princesa Isabel de Bohemia (cf. AT IV, pp. 293-294; Descartes, R.: Correspondencia con Isabel de Bohemia y otras cartas, op. cit., pp. 104-105), e incluso lo asevera en las Meditaciones metafísicas (cf. AT VII, p. 83; Descartes, R.: Meditaciones metafísicas, op. cit., p. 241), igual que en muchos otros pasajes (cf. Shapiro, L.: "The Health of the Body-Machine? Or Seventeenth Century Mechanism and the Concept of Health", Perspectives on Science, 11/4, 2003, p. 424).

42 Al respecto puede leerse un enfático pasaje cartesiano, $c f$. AT IV, pp. 201-202; Descartes, R.: Correspondencia con Isabel de Bohemia y otras cartas, op. cit., p. 63 y, por parte de Leibniz, igualmente, sus declaraciones en cf. A VI. 6, pp. 166-167; Leibniz, G. W.: Nuevos ensayos sobre el entendimiento, op. cit., pp. 236-237. Por supuesto, las diferencias entre el pensamiento cartesiano, o leibniziano, y el estoico son profundas, pues ni el mecanicismo moderno es compatible con la física concebida en el seno del estoicismo que, por ejemplo en el caso de Panecio, concebía el cosmos como un organismo vivo (cf. Rist, J. M.: La filosofía estoica, trad. David Casacuberta, Barcelona, Crítica, 1995, p. 186), ni la concepción fatalista o determinista de los pensadores del período helenístico coincide con la defensa del voluntarismo cartesiano, ni con el trasfondo cristiano de la Modernidad. Ni siquiera la moral cartesiana, pese a lo que aseveran las interpretaciones más usuales, puede entenderse claramente como inspirada por el neo-estoicismo vigente en su época, con eximios representantes como Lipsio o Du Vair. Pues, entre otras cosas, en su tratamiento de la virtud y las pasiones el pensador francés reconoce la existencia de bienes morales, del cuerpo y exteriores que no tolerarían los pensadores estoicos clásicos, ni sus modernos herederos neo-estoicos ( $c f$. Rutheford, D. "Reading Descartes as a Stoic. Appropriate Action, Virtue, and the Passions", Philosophie antique, 14, 2014, pp. 142s).

$43 \quad$ Cf. AT IV, p. 538.

$44 \quad C f$. A VI. 6, pp. 185s; Leibniz, G. W.: Nuevos ensayos sobre el entendimiento, op. cit., pp. 261s. 
trata de obtener ésta, como la finalidad más importante de nuestra vida, mediante un cultivo y contrapeso de las pasiones, a través del cuidado y la atención. Sin embargo, y aunque en sus propuestas morales y terapéuticas son similares, e incluso conciben ambos la felicidad como un placer continuado, difieren con nitidez en la forma de alcanzar esta beatitud terrena.

De este modo, para el pensador francés el modelo clásico de vida feliz, heredado de Aristóteles, especialmente a partir del libro X de la Ética nicomáquea y de los capítulos 1 y 2 del libro A de la Metafísica ya no tendría validez..$^{45}$ Aun cuando con oscilaciones, no es el ejercicio del intelecto, que para Aristóteles se identificaba con la contemplación de lo divino y que conducía a la mejor vida posible, el camino seguro a la felicidad. En efecto, mientras que desde el escolasticismo aristotélico sabiduría y felicidad se entendían como equivalentes, ya desde las Reglas para la dirección del espíritu el pensador francés cuestionó dicha equiparación. ${ }^{46}$ Ciertamente es el ejercicio pleno de la luz natural, esto es, en términos cartesianos, gozar de buen sentido y juzgar correctamente, lo que se entiende por sabiduría y no el conocimiento de un objeto privilegiado, en este caso la divinidad. ${ }^{47} \mathrm{Y}$ este buen sentido se complementa además con la virtud-pasión principal del ser humano, de acuerdo con Las pasiones del alma, a saber, la generosidad, que se basa en el buen uso de nuestro libre arbitrio. ${ }^{48}$ En suma, para ser feliz no basta con conocer bien y juzgar correctamente, sino que también es necesario tener firmeza para ejecutar lo juzgado como mejor y emplear de la manera más adecuada posible dicha facultad de conocer.

Así pues, la felicidad no consiste para Descartes en la aspiración a un conocimiento que nos asimile a la divinidad, puesto que dicho deseo es imposible de satisfacer $y$, en realidad, puede ir en contravía del buen uso de nuestras capacidades. En lugar de esto, en el ámbito práctico, el pensador francés propone antes bien la moderación y el uso prudencial de nuestras facultades. No se trata de pensar con mayor detenimiento, sino como le participa en una importante misiva a la reina Cristina de Suecia del 20 de noviembre de $1647,{ }^{49}$ lo que se deben limitar son nuestros deseos, exactamente a la medida de nuestras finitas capacidades, y hacer bueno uso de las pasiones, para gozar de un moderado contento en nuestra vida. En definitiva, antes que conocer a Dios, o asemejársele, de lo que se trata es de amarle. O por decirlo en términos actuales, es la regulación de nuestra voluntad y no el poder de nuestro intelecto el que nos garantizará la beatitud terrena.

Para Leibniz, por el contrario, la felicidad se equipara, como en el caso de Aristóteles, no sólo con la finalidad de la vida sino también con la perfección, y por

45 Cf. Renault, L.: Descartes ou la félicité volontaire, Paris, Presses universitaires de France, 2000, pp. 17s. De nuevo es importante matizar la oposición cartesiana al aristotelismo, puesto que en diversos pasajes de su obra ética Aristóteles propone que la actividad intelectual es un bien intrínseco entre otros muchos, como la amistad, por ejemplo. De hecho, algunos autores han planteado que la noción intelectualista de la vida feliz encaja con dificultad en la concepción de la eudaimonia que, en general, tuvo el pensador griego, $c f$. Nussbaum, M. C., $L a$ fragilidad del bien. Fortuna y ética en la tragedia y la filosofía griega, trad. de Antonio Ballesteros, Madrid, Machado Libros, 2015, pp. 463s.

46 Cf. AT X, p. 361; Descartes, R.: Reglas para la dirección del espíritu, trad. de Juan Manuel Navarro, Madrid, Alianza, 2003, p. 65.

47 Cf. AT VI, pp. 1-2; Descartes, R.: Discurso del método, trad. de Eduardo Bello, Madrid, Tecnos, 1994, pp. 3-4.

48 Cf. AT XI, pp. 445-446; Descartes. R.: Las pasiones del alma, op. cit., § 153 y Renault, L.: Descartes ou la félicité volontaire, op. cit., p. 47.

$49 \quad C f$. AT V, p. 82. 
una que se alcanza por vía intelectual: "La alegría es un placer que el alma siente en sí misma. El placer es la sensación de una perfección o excelencia en nosotros o en otra cosa". ${ }^{50}$ En efecto, como nos indica el discípulo de Platón, la felicidad es una de las causas finales de la razón, un apetito innato del alma racional $^{51}$ y la misma concepción parece sustentarse en la definición leibniziana de la vida feliz, que no sólo es la duraderamente placentera, sino también la más armoniosa. De lo que se trata, de acuerdo con el pensador alemán, es de pensar la armonía, ${ }^{52}$ es decir, de emplear el intelecto de la mejor manera posible, obrando de acuerdo con las razones óptimas y no arrastrado por los impulsos pasionales, de carácter puntual y confuso. En ambos casos, en el de los afectos y en el de las razones, el objetivo es evitar el dolor y obtener placer, pero mientras que en un caso este último se agota con la consecución de lo placentero, en el otro perdura como sensación de perfección. ${ }^{53}$

No es que el pensador germano desdeñe, claro ésta, el papel de la voluntad en la vida feliz, pero a diferencia de lo que sucede en el caso de Descartes, las pasiones son valoradas de una manera menos positiva, como tendencias necesarias del dinamismo de la vida mental, pero que han de someterse al dominio de la razón. No en vano Leibniz habla de una "ciencia de la felicidad", ${ }^{4}$ puesto que, en su caso, como en el del modelo clásico aristotélico, de lo que se trata es de conocer las causas del placer duradero, es decir, las cualidades que lo hacen posible y perdurable. Por ello, las recomendaciones leibnizianas son claramente intelectualistas y apuntan a una moderación racional de los afectos que contrasta con el voluntarismo cartesiano, pese a que sus concepciones en el ámbito pasional sean tan sorprendentemente cercanas.

\section{Conclusión}

Frente a la interpretación usual de la relación entre el pensamiento de Descartes y el de Leibniz, en este artículo hemos tratado de dejar claro, al menos en relación con el análisis de las emociones, que ambos pensadores tienen mucho más en común de lo que se suele imaginar. Es cierto que la propuesta moral del pensador francés es voluntarista y la del germano intelectualista, ${ }^{55}$ pero esta diferencia es menor si atendemos a sus semejanzas. Tanto Descartes como Leibniz caracterizan las pasiones como elementos de nuestra vida mental de tipo cognitivo y conativo, con una importante dimensión inconsciente, y relacionadas con las opiniones, pero sin identificarse con ellas.

Esto último es importante, porque en su dinamismo dichas tendencias se revelan no sólo necesarias, frente al ideal estoico de la eliminación de las pasiones, caracterizadas como errores del juicio, ${ }^{56}$ sino también inestimablemente suficientes

50 A VI. 5, p. 1302; Leibniz, G. W.: Escritos filosóficos, trad. de Ezequiel de Olaso, Roberto Torreti y Tomás E. Zwanck, Buenos Aires, Charcas, 1982, p. 396.

${ }_{51}$ Cf. Aristóteles: Metafisica, trad. de Tomás Calvo, Madrid, Gredos, 1994, $1013 b 5$.

52 Cf. A VI. 3, p. 116; Leibniz, G. W.: Escritos filosóficos, op. cit., p. 98.

53 Cf. A VI. 6, p. 194; Leibniz, G. W.: Nuevos ensayos sobre el entendimiento, op. cit., p. 269.

${ }^{54}$ A VI. 5, p. 1302; Leibniz, G. W.: Escritos filosóficos, op. cit., p. 396.

55 Losonsky, M.: Enlightenment and Action from Descartes to Kant: Passionate Thought, Cambridge, Cambridge University Press, 2001, pp. 12s.

56 Al respecto puede consultarse con provecho Inwood, B.: Ethics and Human Action in Early Stoicism, Oxford, Clarendon Press, 1985, pp. 175ss, así como una inescapable referencia clásica, la de Séneca: Sobre la ira, trad. de Francisco Navarro, San Cristóbal de la Laguna, Artemisa ediciones, 2011, II, 2, 1 y II, 3, 4. 
para señalarnos el camino a la beatitud humana. En el caso cartesiano mediante su regulación y contrapeso, con el concurso de la voluntad, en el leibniziano pensando más y moderándolas así a través del recto ejercicio del intelecto. En ambos pensadores, pues, los afectos son parte inexcusable de nuestra vida y cabe concluir que para ninguno de los dos habrá nunca demasiada felicidad sin la intervención constante de la filosofía.

\section{Referencias bibliográficas}

Abbagnano, N.: Historia de la filosofía, vol. 2, trad. de Juan Estelrich y Jorge Pérez, Barcelona, Hora, 1994.

Aristóteles: Acerca del alma, trad. de Tomás Calvo, Madrid, Gredos, 1978.

Aristóteles: Ética nicomáquea. Ética eudemia, trad. de Julio Pallí, Madrid, Gredos, 1985.

Aristóteles: Metafísica, trad. de Tomás Calvo, Madrid, Gredos, 1994.

Aristóteles: Partes de los animales. Marcha de los animales. Movimiento de los animales, trad. de Elvira Jiménez y Almudena Alonso, Madrid, Gredos, 2000.

Belaval, Y.: Leibniz, Critique de Descartes, Paris, Gallimard, 1960.

Brown, D. J.: Descartes and the Passionate Mind, Cambridge, Cambridge University Press, 2006.

Brown, D. J.: "The Sixth Meditation: Descartes and the Embodied Self", en D. Cunning (ed.), The Cambridge Companion to Descartes' Meditations, Cambridge, Cambridge University Press, 2014, pp. 240-257.

Damasio, A. R.: Descartes' error. Emotion, Reason, and the Human Brain, New York, Avon Books, 1995.

Descartes, R.: El tratado del hombre, trad. de Guillermo Quintás, Madrid, Alianza, 1990.

Descartes, R.: Discurso del método, trad. de Eduardo Bello, Madrid, Tecnos, 1994.

Descartes, R.: Euvres complètes, Paris, Vrin, 1996.

Descartes, R.: Correspondencia con Isabel de Bohemia y otras cartas, trad. de Maite Gallego, Barcelona, Alba, 1999.

Descartes, R.: Reglas para la dirección del espíritu, trad. de Juan Manuel Navarro, Madrid, Alianza, 2003.

Descartes, R.: Meditaciones metafisicas, trad. de Vidal Peña, Oviedo, KrK, 2005.

Descartes. R.: Las pasiones del alma, trad. de José Antonio Martínez y Pilar Andrade, Madrid, Tecnos, 2006.

Di Bella, S.: "Le De affectibus leibnizien: de la dynamique des passions à la constitution de la substance individuelle", en P.-F. Moreau (ed.), Les passions à l'âge classique. Théorie et critiques des passions, vol. 2, Paris, Presses universitaires de France, 2006, pp. 193-208.

Ebbersmeyer, S.: "Leibniz on the Passions and the Dynamical Dimension of the Human Mind”, en S. Ebbersmeyer (ed.), Emotional Minds. The Passions and the Limits of Pure Enquiry in Early Modern Philosophy, Berlin, Boston, De Gruyter, 2012, pp. 139-159.

Gaukroger, S.: Descartes. An Intellectual Biography, Oxford, Clarendon Press, 1995.

Gaukroger, S.: Descartes' System of Natural Philosophy, Cambridge, Cambridge University Press, 2002.

González Recio, J. L. (ed.): La correspondencia Descartes-Henry More, trad. de Dolores Escarpa e Irene Pajón, Madrid, Antígona, 2011.

Inwood, B.: Ethics and Human Action in Early Stoicism, Oxford, Clarendon Press, 1985.

Kulstad, M.: "Leibniz's concept of expression”, Studia Leibnitiana, 11/1, 1977, pp. 57-76. 
Leibniz, G. W.: Sämtliche Schriften und Briefe, Darmstadt y Berlin, Academia alemana de ciencia, 1923-.

Leibniz, G. W.: Monadología, trad. de Julián Velarde, Oviedo, Pentalfa, 1981.

Leibniz, G. W.: Escritos filosóficos, trad. de Ezequiel de Olaso, Roberto Torreti y Tomás E. Zwanck, Buenos Aires, Charcas, 1982.

Leibniz, G. W.: Nuevos ensayos sobre el entendimiento, trad. de Juan R. Goberna, Madrid, Akal, 2016.

Losonsky, M.: Enlightenment and Action from Descartes to Kant: Passionate Thought, Cambridge, Cambridge University Press, 2001.

Marion, J.-L.: Sobre la ontología gris de Descartes, trad. Alejandro García Mayo, Madrid, Escolar y Mayo Editores, 2008.

Nussbaum, M. C., La fragilidad del bien. Fortuna y ética en la tragedia y la filosofía griega, trad. de Antonio Ballesteros, Madrid, Machado Libros, 2015.

Reale, G. y Antiseri, D.: Historia del pensamiento filosófico y científico, vol. 2, trad. de Juan Andrés Iglesias, Barcelona, Herder, 1992.

Renault, L.: Descartes ou la félicité volontaire, Paris, Presses universitaires de France, 2000.

Rist, J. M.: La filosofía estoica, trad. David Casacuberta, Barcelona, Crítica, 1995.

Rodis-Lewis, G.: Le problème de l'inconscient et le cartésianisme, Paris, Presses universitaires de France, 1950.

Rutheford, D. "Reading Descartes as a Stoic. Appropriate Action, Virtue, and the Passions", Philosophie antique, 14, 2014, pp. 129-155.

Séneca: Sobre la ira, trad. de Francisco Navarro, San Cristóbal de la Laguna, Artemisa ediciones, 2011.

Shapiro, L.: "The Health of the Body-Machine? Or Seventeenth Century Mechanism and the Concept of Health", Perspectives on Science, 11/4, 2003, pp. 421-442. Doi: https://doi. org/10.1162/106361403773082252.

Shorto, R.: Los huesos de Descartes, trad. de Claudia Conde, Barcelona, Planeta, 2011.

Simmons, A.: "Changing the Cartesian Mind: Leibniz on Sensation, Representation and Consciousness", The Philosophical Review, 110/1, 2001, pp. 31-75.

Simmons, A.: "Mind-Body Union and the Limits of Cartesian Metaphysics", Philosophers' Imprint, 17/14, 2017, pp. 1-36.

Spinoza, B.: Spinoza Opera, vol. 2, Heidelberg, Carl Winters Universitätsbuchhandlung, 1972.

Spinoza, B.: Ética demostrada según el orden geométrico, trad. de Vidal Peña, Madrid, Tecnos, 2007.

Swoyer, Ch.: "Leibnizian expression", Journal of the history of philosophy, 33/1, 1995, pp. 65-99.

Vargas, E.: "Perceiving machines: Leibniz's teleological approach to perception", en J. E. H. Smith y O. Nachtomy (eds.), Machines of nature and corporeal substances in Leibniz, Dordrecht, Springer, 2011, pp. 175-186.

Williams, B.: Descartes: The Project of Pure Enquiry, London, Routledge, 2005. 